\title{
Directed metabolomic approaches for the characterization and development of new yeast strains
}

\author{
Ignacio Belda ${ }^{1}$, Santiago Benito ${ }^{2}$, Javier Ruiz ${ }^{1}$, Lorena B. Conchillo ${ }^{1}$, Alejandro Alonso ${ }^{1}$, Domingo Marquina ${ }^{1}$ \\ Fernando Calderón ${ }^{2}$, Eva Navascués ${ }^{1,3}$, and Antonio Santos ${ }^{1}$ \\ ${ }^{1}$ Department of Microbiology, Biology Faculty, Complutense University of Madrid, 28040 Madrid, Spain \\ ${ }^{2}$ Department of Chemistry and Food Technology, Polytechnic University of Madrid, 28040 Madrid, Spain \\ ${ }^{3}$ Agrovin S.A., 13600 Alcázar de San Juan, Spain
}

\begin{abstract}
Analyzing the influence of different yeast species on several compounds with enological interest, it becomes possible to identify metabolic determinants of the incidence of yeasts on wine quality. Contrary to Saccharomyces cerevisiae, understanding genetic regulation, enzymatic properties and physiology of non-Saccharomyces species in enological conditions is far from being known. Because of this, the commercialization of industrial non-Saccharomyces strains on wine industry is showing a really slow pace. In order to determine the enzymatic properties of wine-related yeast species it is necessary to evaluate hundreds of yeast isolates enabling us to robustly attribute specific enzymatic activities to a specific group of yeast species. The contribution of yeasts to wine flavour is greatly determined by their impact on aromatic compounds release. Different glycosidases, $\beta$-lyase, pectinase, cellulase and protease activities are described as responsible for changes in wine composition, so determining inter- and intraspecific variability in these enzymatic properties in yeast species seems to be a useful tool for innovative yeast selection process. With the aim of relating enzymatic activities with a specific impact in wine properties we developed combined fermentations with non-Saccharomyces selected strains and industrial S. cerevisiae strains. The use of rational metabolomic analysis allows us to explain the physiology of non-Saccharomyces yeasts during wine fermentation and its incidence on wine quality.
\end{abstract}

\section{Introduction}

New analytical methodologies have revolutionized research on wine microbiology through the development of metabolic studies that allow us to understand the physiology of new yeast strains. During the past decades, most yeast distribution companies invested their efforts and high amounts of money in the isolation and characterization of different and innovative $S$. cerevisiae strains. This fact has enabled winemakers to elaborate their own genuine wines, flexibly adapted to cellar environments and product styles.

Nowadays, winemaking trends are laid down by the likes of consumers, increasingly demanding in terms of sensorial quality and food safety. In this context, the ability to adapt quickly to changes in consumer's tastes or even anticipate them, seems to be the key to success for the future of enology industry.

Viticulture has manned much of the work in offering a great variety of wines, having nearly 326 red grape varieties and 323 white varieties with their respective selected clones. This variety is now appreciated and known by consumers, so it is time to establish a new change that must happen inside the cellar, through an exhaustive knowledge of the entire fermentation process and the possibilities of flavour modulation that it offers.

The targets of enological microbiology can be channeled by three different focuses: sensitive, technological and fermentative properties of the microbial strains. Enzymatic properties of different microorganisms involved in winemaking process (bacteria and yeasts, mainly) have been studied for a long time [1], but yeasts have featured this applied research line as the main microorganism responsible of the alcoholic fermentation process [2-5].

Many research groups are currently studying nonSaccharomyces yeasts [3,6-10] due to their unique physiological properties, which may be useful in innovative winemaking. The presence of non-Saccharomyces wild yeasts in fermentations has been associated, traditionally, with high levels of acetic acid and other off-flavours. Nevertheless, nowadays researchers and winemakers are aware of the influence of non-Saccharomyces in wine aroma complexity [11-20].

The difficulty of non-Saccharomyces wine yeast to finish the alcoholic fermentation requires the development of combined or multistarter fermentation with $S$. cerevisiae as a binding partner. Some enzymatic properties (glycosidases, $\beta$-lyase, etc.) and the release of some interesting metabolites such as glycerol, mannoproteins and other aromatic compounds justify the interest in these combined fermentations [21,22]. Such fermentations are a very useful tool to improve wine productions in which aromatic complexity of spontaneous fermentations and the safety of industrial targeted fermentations are joined [19,21].

The scientific efforts to understand the metabolism of non-Saccharomyces wine yeasts allow to avoid the 
exclusively empirical knowledge about the influence of these yeasts in wine composition and flavour.

The use of metabolomics in wine fermentations enables to describe the physiology of different yeast strains and to determine its influence on wine quality. This is an interesting operating mode but usually offers biased information as a function of fermentation conditions (grape variety, fermentation temperature, etc.). The study of the enzyme potential of yeasts, studied at a genetic or enzymatic level in vitro, turns this perspective allowing the targeted use of yeasts with specific enzyme activities for the study of their direct influence on the release of aromatic compounds.

The aim of this work is to develop an enzymatic study in a large collection of yeasts that will allow us to understand the variety and richness in hydrolytic enzymes directly derived from the grape must yeast population. This basic knowledge is then applied to prove the repercussion of these enzymes and therefore of the yeast strains on the wine composition through the release of aromatics, phenolics and other colour compounds.

In order to bridge the gap between the laboratory and the industry scale, promising results with microvinifications should be tested at an industrial scale. This fact is one of the bottlenecks of the translational research in applied microbiology due to the influence of the scale on yeast gene expression and, in consequence, on yeast physiology [23].

This work has been divided into 5 items: a) Isolation of a great variety of yeast strains from different vineyards; b) Molecular identification of the isolated yeast strains; c) Characterization of their enzymatic properties (glycosidases, $\beta$-lyase, pectinases, protease, etc.); d) Study, at a laboratory scale (microvinifications), of the enological properties of the selected yeast strains; e) Development of industrial fermentations with successful yeast strains to validate their industrial applicability.

\section{The importance of a proper isolation process}

Grape must enables the growth of several microorganisms, specially yeasts and fungi. Current taxonomies recognize 149 yeast genera comprising nearly 1500 species [24]. Of these, more than 40 species have been isolated from grape must $[3,21]$ but not all of them are welcome to the fermentation process. It should be noted that some yeast species found in grapes and must, like Aureobasidium pullulans or Cryptococcus amylolentus, are enzymatically interesting showing a wide range and intensity of hydrolytic activities but have a low incidence on fermentation development. A. pullulans dominates the microbial consortia of grape [25,26], however, its null fermentative power and its low adaptation to fermentative environment bias their contribution to the fermentation process, making their interest in winemaking scarce [27]. There is a simple method to minimize the isolation of this yeast-like fungus by keeping the fresh grape must refrigerated overnight in order to greatly reduce the Aureobasidium population.

After this, the use of a selective medium for nonSaccharomyces is required in order to avoid the isolation of $S$. cerevisiae strains. S. cerevisiae is not the most abundant species in fresh grape must [28], but depending on the origin, significant amounts of $S$. cerevisiae strains can be found [29]. The use of "lysine medium" containing lysine as the only nitrogen source inhibits the growth of $S$. cerevisiae, which is unable to use lysine as only nitrogen source [30].

\section{Population study. Rational use of genomic technology}

There is a growing trend in the use of "omic" technologies for the holistic approach of population studies. Classical molecular technics like t-RFLP [31,32] or DGGE [25], genomic approaches [28,33,34] and metagenomic tools $[26,35]$ are culture independent techniques, but we cannot forget the importance of microorganism cultures for their further study and industrial usage. Knowledge about the vineyard and fermentation microbiomes are growing notably in recent years [26,35,36], so now we have quite enough information about yeast abundance and distribution in both grapes and fermentations. However, information about comparative genomics of different yeast strains is scarce, even more in the case of non-Saccharomyces species.

The lack of genomic information about most winerelated yeast species, apart from $S$. cerevisiae [37], entails the difficulty of the application of molecular technics to glimpsing the relative abundance of interesting enzymes through the detection, characterization and quantification of their correspondent genes. Maybe, the coordinated development of knowledge about enzymatic properties and genomics of wine yeast species will allow for the development of "comparative metagenomics" to change the focus of classical phylogenetic studies.

In this study, due to its applied objective, a mixed approach was adopted in order to have population data but keeping a wide collection of yeasts for further characterization.

After isolation and freezing $\left(-80^{\circ} \mathrm{C}\right)$, all yeast isolates were identified by sequence analysis of the $26 \mathrm{~S}$ large subunit rRNA gene [38].

Figure 1 shows that, according to several population studies on grape and must microbiome [4,15,31], Hanseniaspora uvarum was the most abundant nonSaccharomyces yeast found in must, being around a 50\% of the total. Its low fermentative power causes the rapid decrease of $H$. uvarum during the early stages of wine fermentation, so besides its low production of hydrolytic enzymes, its influence on final wine composition is not significant.

The next most abundant species, Metschnikowia pulcherrima and Kluyveromyces thermotolerans, reach $12 \%$ of the total yeast population each. Scientific works about these two species are abundant [7,39-42], being the most common non-Saccharomyces species commercially available for wine industry besides Torulaspora delbrueckii strains $[10,43,44]$. T. delbrueckii showed a low implantation rate in fresh must but, due to its high fermentation power and ethanol tolerance $(9-11 \%)$, it is one of the non-Saccharomyces yeasts with higher incidence on wine properties and quality. 


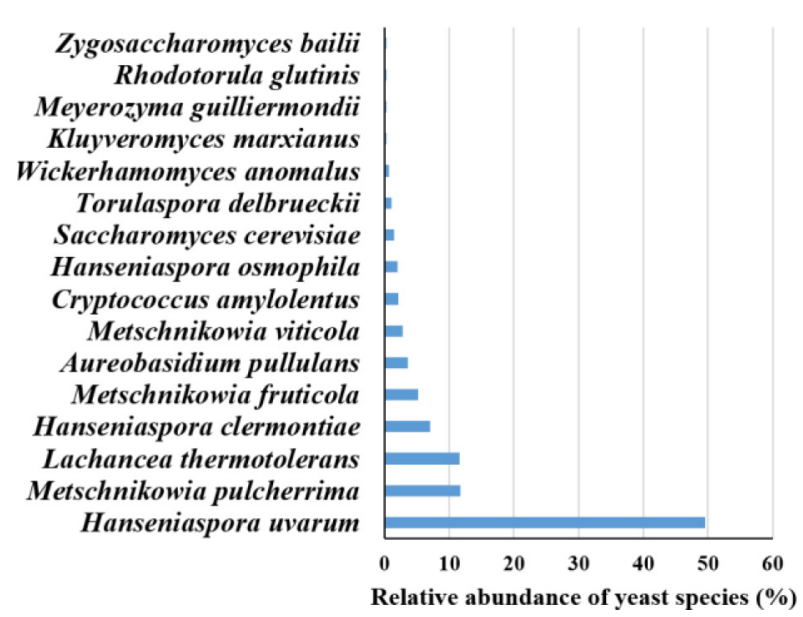

Figure 1. Composition of yeast population comprising the analyzed collection.

Despite the cautions adopted during the isolation process regarding A. pullulans isolates, the percentage of this species reaches $4 \%$ of the total. However, later enzymatic analysis revealed some interesting properties of this species.

It should be noted that within Hanseniaspora and Metschnikowia genera, three different species can be identified, respectively (Fig. 1). Their phylogenetic proximity causes that several metabolic parameters are shared between species on the same genus but, as we can see in our enzymatic assays, each one of them shows different enzymatic profiles.

\section{Enzymatic study. Foreseeing the repercussion of yeasts on wine quality}

One of the most recognized advantages of the use of nonSaccharomyces as an inoculum for wine fermentation is the production of a great variety of hydrolytic enzymes. This contributes widely to wine complexity complementing the incidence of $S$. cerevisiae in aromatic compounds release [21].

The importance of terpenes in some wines is well known, being the main aromatic descriptors of Vitis vinifera var. Muscat, Riesling or Albariño [45]. Grape varieties contain small amounts of free terpenes but in all cases a notably proportion of the total are conjugated to sugar residues forming glycoconjugated compounds [46].

Terpene glycosides can be hydrolyzed in an enzymatic way by the action of glycosidases [47]. The enzymatic hydrolysis of glycosides requires the sequential action of two enzymes in two separate steps: firstly, $\beta$-D-xylosidase, $\alpha-\mathrm{L}$ arabinofuranosidase, $\alpha$-L-rhamnosidase or $\beta$-D-apiosidase cleavage the terminal sugar releasing xylose, arabinose, rhamnose or apiose. Then, liberation of monoterpenol from the free $\beta$-glucoside takes place after action of a $\beta$-Dglucosidase (Fig. 2).
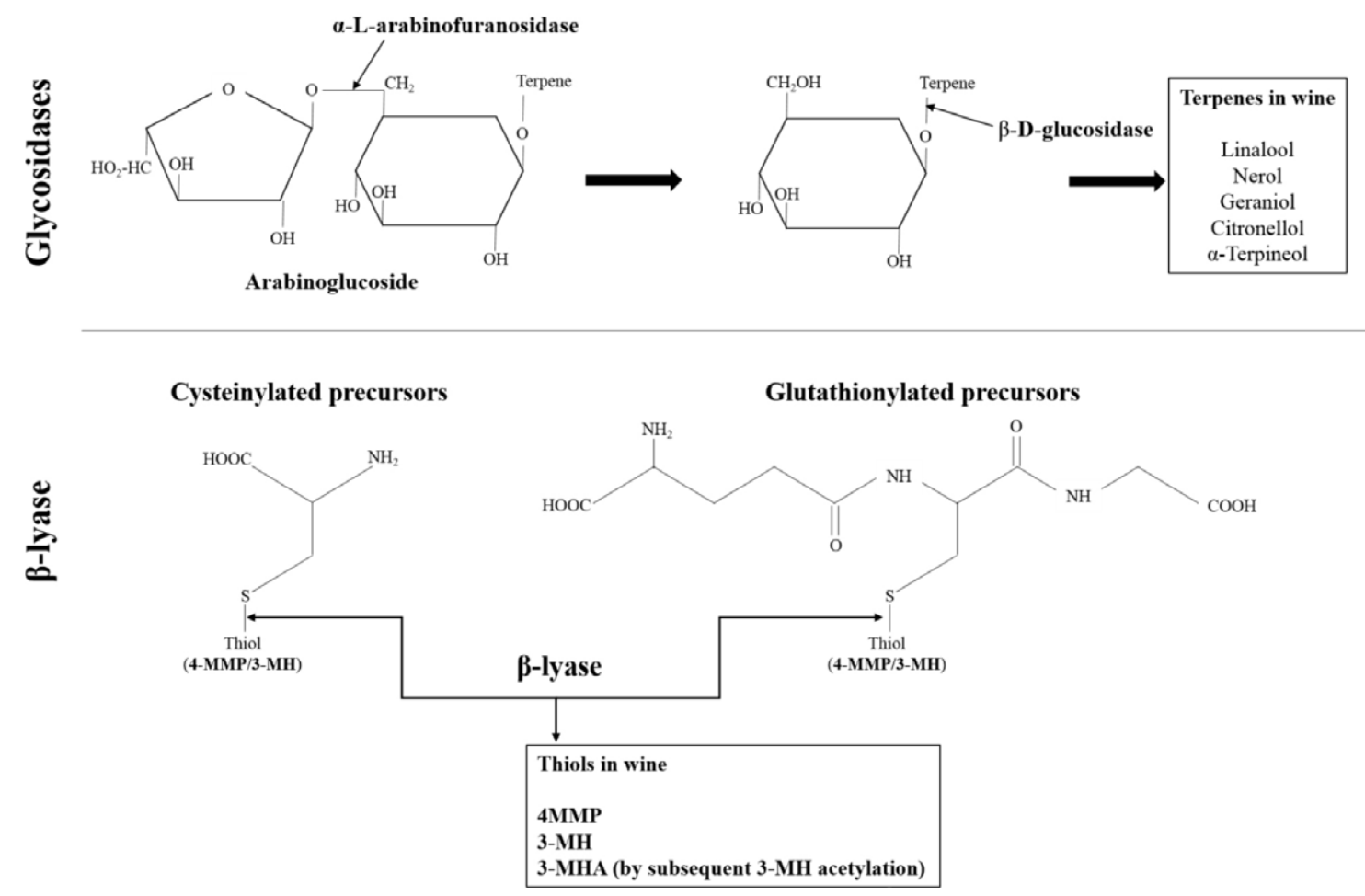

Figure 2. Enzymatic release of terpenes and thiols in wines. 
Although some $S$. cerevisiae strains have certain glycosidases, such as xylosidase, most of them have no $\beta$-D-glucosidase activity $[48,49]$, which is necessary to release the terpene from its glucoside. In this context, non-Saccharomyces yeasts can provide $\beta$-D-glucosidase enzymes to complete the aroma release. Most of nonSaccharomyces yeasts have this activity, in addition to high levels of $\beta$-D-xylosidase, $\alpha$-L-arabinofuranosidase, $\alpha$-L-rhamnosidase or $\beta$-D-apiosidase, so their use seems to be an adequate tool for the aromatic enhancement in terpenic grape varieties and also for aroma release in neutral varieties.

In this study, most of Hanseniaspora uvarum strains showed a great $\beta$-D-glucosidase activity, according to previous works where $H$. uvarum was confirmed to be able to release terpenols in wine [50,51]. Our experiments also showed that in vitro $M$. puclherrima has a notable $\beta$-D-glucosidase, which was deployed in all isolated strains and, again, its applicability in terpene release during wine fermentation has been proved [51].

Some industrial M. pulcherrima strains are described as useful for aromatic enhancement of wines due to their $\alpha$-L-arabinofuranosidase activity [4], but this activity is really limited to a few strains. However, $\beta$-D-xylosidase was highly present in most $M$. pulcherrima isolated strains in this work.

Volatile thiols are aromatic compounds of particular importance to the varietal character, imparting passionfruit, grapefruit, box tree aroma at appropriate concentrations. 4-mercapto-4-methylpentan-2-one (4MMP), 3-mercaptohexan-1-ol (3MH) and 3-mercaptohexyl acetate (3MHA) are the aromatic thiols found in wines, being the main descriptors of grape varieties such as Sauvignon blanc or Verdejo [52]. The low sensory threshold of these compounds makes them one of the main objectives in wine improvement through aromatic compounds release [53]. These volatile thiols are virtually non-existent in grape juice and only released during fermentation. Certain $S$. cerevisiae strains are able to release these thiols from their conjugated form (cysteinylated or glutathionylated precursors) due to their $\beta$-lyase activity (Fig. 2). 4MMP and $3 \mathrm{MH}$ are formed from grape-derived non-volatile conjugates and yeasts are simply involved in the cleaving of the thiol from the precursor. Nevertheless, even the best S. cerevisiae producer rarely transforms more than $5 \%$ of the cysteinylated precursor (the most abundant conjugated form in must) initially contained in the must into thiols [54].

This limitation justifies searching for alternative yeast species able to release higher amounts of these thiols.

Our enzymatic screening and, also our later vinification assays, suggest that $T$. delbrueckii are one of the most efficient species regarding $\beta$-lyase activity, in both, in vitro and fermentation assays showing that most strains have a great capability for thiol release from its cysteinylated precursor.

These latter two activities (glycosidases and $\beta$-lyase) are directly related with aroma release. However, other enzymatic activities with other enological applications that have been long-time used in industry (presented as enzymatic preparations of fungal origin) can be found in different yeast species.

Pectinolytic enzymes are widely used in enology helping to the degradation of polysaccharides comprising the vegetal wall of grape's skin and pulp (Fig. 3). They can help to improve clarification and filterability processes, releasing more colour and flavour compounds entrapped in the grape skin and facilitating the liberation of phenolic compounds [1]. Traditionally, commercial pectinases used in winemaking comprise the mixtures of polygalacturonase, pectate lyase and pectin methylesterase enzymes [55]. Of these, two types of polygalacturonases, endo- and exopolygalacturonase, are mainly responsible for pectinolytic activity and hence are enzymes of particular importance to the industries [56].

Our results showed that polygalacturonase enzymes are scarce on wine yeasts species. Noting that some $S$. cerevisiae strains has polygalacturonase, most of them have no activity or are not efficient enough in fermentation conditions to avoid clarification and filtration problems [2]. Only A. pullulans and some species of the genus Metschnikowia (M. pulcherrima and M. fruticola) showed polygalacturonase activity in vitro and our fermentation assays suggest that only the activity of M. pulcherrima can be considered for its application in industry.

The effect of temperature on the efficiency of pectinolytic activity has been studied (data not shown) suggesting that pre-fermentative cold soak is a great method to enhance the incidence of these enzymes on wine composition and quality.

In the same way, cellulase enzymes work on the improvement of maceration and filterability processes. This enzymatic activity is rarely found in yeasts, only showed by A. pullulans strains. At the moment and, based on our results, yeasts cannot be considered as a source of cellulases for enological application.

Finally, we analyzed the presence of protease enzymes in our yeast collection, concluding that this activity is absent in $S$. cerevisiae and also in other species like T. delbrueckii, Metschnikowia viticola, Lachancea thermotolerans, Zygosaccharomyces bailii and Cryptococcus amylolentus being widely present in the other studied yeast species.

The use of proteases in winemaking is not a really extended practice at the moment, being more frequent the use of bentonite to solve protein haze problems. The use of bentonite usually causes an impoverishment of sensorial properties of wines, so the use of proteases for this objective seems to be a future real solution [57].

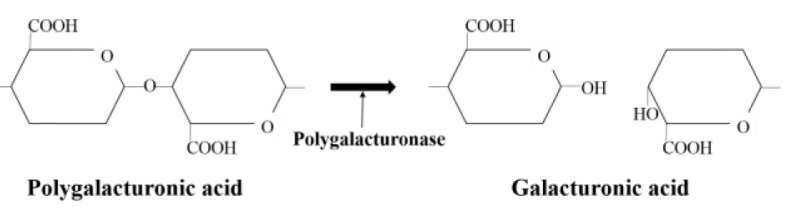

Figure 3. Enzymatic degradation of pectic polysaccharydes (polygalacturonic acid) from grape skin and pulp. 


\section{Microvinifications: A model medium for physiological studies of wine yeasts depending on the approval of industrial scale}

\subsection{The influence of Torulaspora delbrueckii on wine composition and quality}

T. delbrueckii is, at present, the most applied non-Saccharomyces yeast in the wine industry. Most of yeast distribution companies have developed T. delbrueckii strains and work, together with researchers, in the gain of knowledge about their physiology and genetic and metabolic regulation in fermentation conditions [10,43,44,58,59].

In the present study, the physiology of $T$. delbrueckii during red wine fermentations in both, sequential and simultaneous inoculation with $S$. cerevisiae CT007 (Agrovin S.A.), was analyzed. First of all, the implantation rate of the studied strain ( $T$. delbrueckii NS-TD, Agrovin S.A.) was notable. It was detected during the first three quarters of the process, when it was used by sequential inoculation (17 days of a total of 22 days of fermentation) and during the first half of the fermentation with simultaneous inoculation ( 7 days of a total of 14) at a microvinification scale. In the case of industrial fermentation, the delay detected in the fermentation process in the sequential inoculation was lower than in laboratory assays, detecting a slight delay at the start of fermentation, but no significant differences in finishing the fermentation.

The most noteworthy aspects of the impact of T. delbrueckii on wine composition are its repercussion on wine quality due to its incidence on the ethanol concentration that is slightly reduced, the release of higher levels of glycerol and pyruvic acid and the fewer amounts of higher alcohols released. It is also worth mentioning the repercussion of $T$. delbrueckii on the structure of wines, which has been explained by its large release of mannoproteins (Fig. 4).

Because of this, a better mouthfeel structure of sequential fermentations that significantly contributed to the

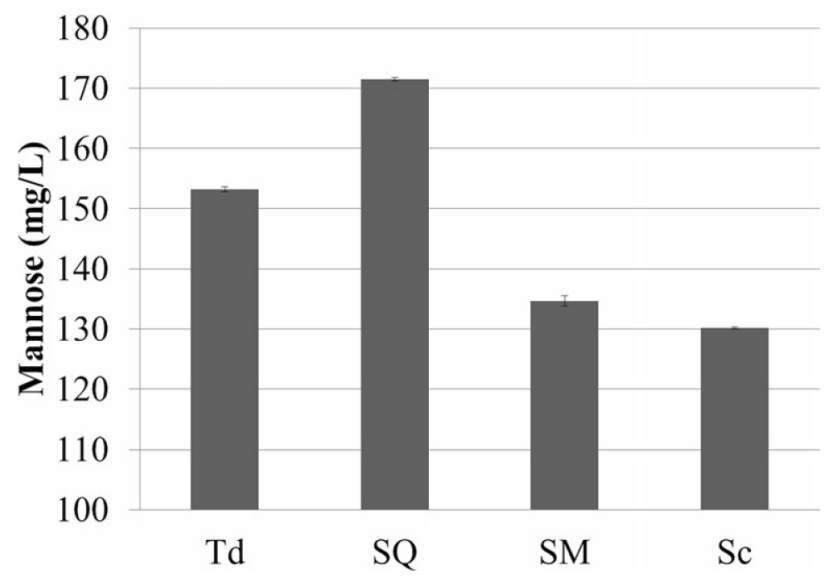

Figure 4. Mannoprotein content of wines fermented at semiindustrial scale with: S. cerevisiae CT007 (Sc); T. delbrueckii NS-TD and $S$. cerevisiae CT007 by using simultaneous inoculation (SM); sequential inoculation (SQ); and T. delbrueckii NS-TD alone (Td). Adapted from Belda et al. (2015) [10]. higher overall score was obtained in the sensorial analysis of these wines (Fig. 5). In addition, a higher perception in sweetness was detected, probably due to the malic acidity consumed by T. delbrueckii, but also influenced by higher levels of mannoproteins in wines.

\subsection{Application of selected M. pulcherrima strain based on their pectinolytic activity}

Apart from T. delbrueckii, M. pulcherrima is one of the most commonly used non-Saccharomyces yeasts in enology, being, additionally, one of the most widely distributed yeasts in spontaneous fermentations. The information about its physiology in fermentation is lower than in the case of $T$. delbrueckii, but researchers are starting to be very interested in its metabolism and its different applicability in wine.

One of the main current focuses of research in $M$. pulcherrima wine strains is to reduce the alcohol content of wines because of its lower fermentative efficiency and its respiratory metabolism [7,42]. Another point of interest is the production of pulcherrimin pigment that causes certain antimicrobial effect on the other yeast populations, including the spoilage yeast Brettanomyces bruxellensis [39]. Apart from that, the notable implantation of $M$. pulcherrima during wine fermentation, when it is used in sequential fermentation with $S$. cerevisiae, causes its incidence in volatile compounds of wines producing high concentrations of esters [60,61].

Certain yeast species, such as Rhodotorula mucilaginosa, have been described as polygalacturonase active in enological conditions [62]. In the case of this study, the pectinolytic activity of $M$. puclherrima was analyzed for its application in red wine fermentations in order to improve some sensorial and technological properties, such as colour intensity and turbidity and filterability of wines.

Figure 6 shows that the use of $M$. pulcherrima in sequential fermentation with $S$. cerevisiae causes a significant

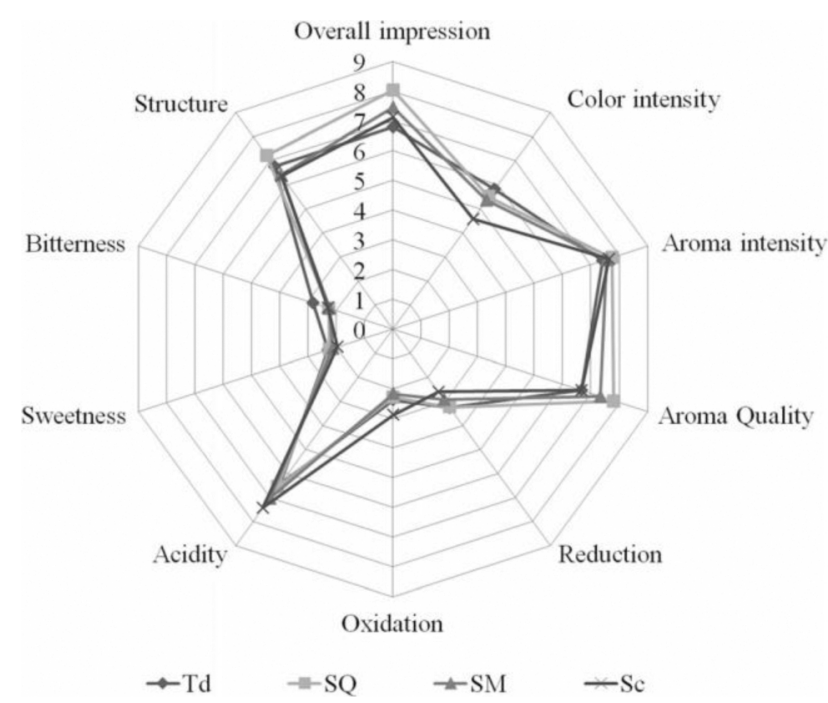

Figure 5. Taste and olfactory attribute scores for the final wines.: S. cerevisiae CT007 (Sc); T. delbrueckii NS-TD and S. cerevisiae CT007 by using simultaneous inoculation (SM); sequential inoculation (SQ); and T. delbrueckii NS-TD alone (Td). Adapted from Belda et al. (2015) [10]. 

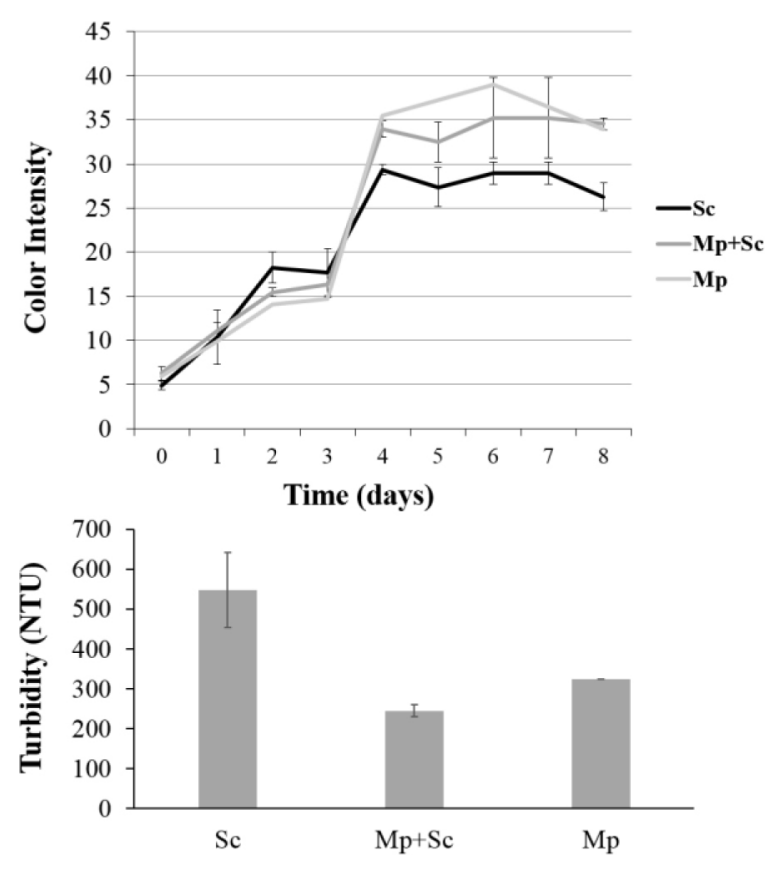

Figure 6. Evolution of colour intensity during fermentation and turbidity of final wines (Nephelometric Turbidity Units) fermented by $S$. cerevisiae alone (Sc); sequential inoculation of M. pulcherrima and S. cerevisiae $(\mathrm{Mp}+\mathrm{Sc}) ;$ M. pulcherrima alone (Mp).

effect in sensorial parameters such as colour intensity and in technological aspects, like turbidity of wines. These results, obtained at a semi-industrial scale, $(700 \mathrm{~kg}$ of $V$. vinifera var. Tempranillo) open a new perspective of application of $M$. pulcherrima strains in winemaking, making necessary the optimization of its use by further characterization of its pectinolytic activity.

\section{Conclusions}

The large distance between the knowledge about $S$. cerevisiae and non-Saccharomyces yeasts as a new world for enology is the main responsible of their low presence as inoculum in cellar. Winemakers are now open to changes in fermentation paradigms but the pillars of this change should be firmly established.

Increasing the number of studies and the knowledge about the potential repercussion of non-Saccharomyces in wine fermentations it is necessary to deep study their genetic and metabolic regulation through the variables existing on the fermentation process. Furthermore, in order to anticipate to market movements and likes, new non-Saccharomyces yeast strains apart from the "classics" (T. delbrueckii, M. pulcherrima, L. thermotolerans and Pichia kluyvery) should be explore.

Microbial richness on vineyard can be directly related to enzymatic diversity on fermentation so, viticulture practices that contribute to microbial diversity (without forgetting the control of spoilage microorganisms) should be greatly considered, avoiding aggressive phytosanitary practices.
The use of yeasts based on theirs enzymatic properties seems to be a useful manner to a targeted use of non-Saccharomyces strains in the correct grape variety, achieving the desired flavor enhancement.

\section{References}

[1] P. Van Rensburg, I.S. Pretorius, S. Afr. J. Enol. Vitic. 21, 52-73 (2000)

[2] I.S. Pretorius, Yeast 16, 675-729 (2000)

[3] N.P. Jolly, O.P.H. Augustyn, I.S. Pretorius, S. Afr. J. Enol. Vitic. 27, 15-39 (2006)

[4] N.P. Jolly, C. Varela, I.S. Pretorius, FEMS Yeast Res. 14, 215-237 (2014)

[5] A.G. Cordente, C.D. Curtin, C. Varela, I.S. Pretorius, Appl. Microbiol. Biotechnol. 96, 601-618 (2012)

[6] F. Comitini, M. Gobbi, P. Domizio, C. Romani, L. Lencioni, I. Mannazzu, M. Ciani, Food Microbiol. 28, 873-882 (2011)

[7] A. Contreras, C. Hidalgo, P.A. Henschke, P.J. Chambers, C. Curtin, C. Varela, Appl. Environ. Microbiol. 80,1670-1678 (2014)

[8] T. Garde-Cerdán, C. Ancín-Azpilicueta, Trends Food Sci. Technol. 17, 438-447 (2006)

[9] A. Alonso, I. Belda, A. Santos, E. Navascués, D. Marquina, Food Control 51, 129-134 (2015)

[10] I. Belda, E. Navascués, D. Marquina, A. Santos, F. Calderón, S. Benito, Appl. Microbiol. Biotechnol. 99, 1911-1922 (2015)

[11] C.N. Egli, W.D. Edinger, C. Mitrakul, T. HenickKling, J. Appl. Microbiol. 85, 779-789 (1998)

[12] B. Esteve-Zarzoso, P. Manzanares, D. Ramón, A. Querol, Int. Microbiol. 1, 143-148 (1998)

[13] G.H. Fleet, Int. J. Food Microbiol. 86, 11-22 (2003)

[14] G.H. Fleet, FEMS Yeast Res. 8, 979-995 (2008)

[15] G.H. Fleet, G.M. Heard, Wine microbiology and biotechnology (Harwood Academic Publishers, Chur, 1993)

[16] J.V. Gil, J.J. Mateo, M. Jimenez, A. Pastor, T. Huerta, J. Food Sci. 61, 1247-1250 (1996)

[17] T. Henick-Kling, W. Edinger, P. Daniel, P. Monk, J. Appl. Microbiol. 84, 865-876 (1998)

[18] M.G. Lambrechts, I.S. Pretorius, S. Afr. J. Enol. Vitic. 21, 97-129 (2000)

[19] P. Romano, C. Fiore, M. Paraggio, M. Caruso, A. Capece, Int. J. Food Microbiol. 86, 169-180 (2003)

[20] F. Viana, J.V. Gil, S. Genovés, S. Vallés, P. Manzanares, Food Microbiol. 25, 778-785 (2008)

[21] M. Ciani, F. Comitini, I. Mannazzu, P. Domizio, FEMS Yeast Res. 10, 123-133 (2010)

[22] V. Rojas, J.V. Gil, F. Piñaga, P. Manzanares, Int. J. Food Microbiol. 70, 283-28 (2001)

[23] D. Rossouw, N.P. Jolly, D. Jacobson, F.F. Bauer, Appl. Microbiol. Biotechnol. 93,1207-1219 (2012)

[24] C.P. Kurtzman, W.F. Fell, T. Boekhout, The Yeasts, a Taxonomic Study, 5th edn. (Elsevier Science Pulishers, Amsterdam 2011)

[25] C.J. Prakitchaiwattana, G.H. Fleet, G.M. Heard, FEMS Yeast Res. 4, 865-877 (2004)

[26] C. Pinto, D. Pinho, S. Sousa, M. Pinheiro, C. Egas, A.C. Gomes, PLOS One 9, e85622 (2014)

[27] V. Renouf, O. Claisse, A. Lonvaud-Funel, Appl. Microb. Cell Physiol. 75, 149-164 (2007) 
[28] M.J. Torija, N. Rozes, M. Poblet, J.M. Guillamon, A. Mas, A. Van Leeuw. J. Microb. 79, 345-352 (2001)

[29] G. Beltrán, M.J. Torija, M. Novo, N. Ferrer, M. Poblet, J.M. Guillamon, N. Rozés, A. Mas, System. Appl. Microbiol. 25, 287-293 (2002)

[30] L.S. Walters, M.R. Thiselton, J. Inst. Brew. 59, 401404 (1953)

[31] N.A. Bokulich, C.F. Hwang, S. Liu, K.L. BoundyMills, D.A. Mills, Am. J. Enol. Vitic. 63, 185-194 (2012)

[32] Y. Sun, Y. Liu, Food Microbiol. 38, 201-207 (2014)

[33] Y.P. Maturano, M.V. Mestre, B. Esteve-Zarzoso, M.C. Nally, M.C. Lerena, M.E. Toro, F. Vazquez, M. Combina, Int. J. Food Microbiol. 199, 23-32 (2015)

[34] N.A. Bokulich, D.A. Mills, Appl. Environ. Microbiol. 79, 2519-2526 (2013)

[35] N.A. Bokulich, J.H. Thorngate, P.M. Richardson, D.A. Mills, PNAS 111, E139-E148 (2014)

[36] I. Zarraonaindia, S.M. Owens, P. Weisenhorn, K. West, J. Hampton-Marcell, S. Lax, N.A. Bokulich, D.A. Mills, G. Martin, S. Taghavi, D. van der Lelie, J.A. Gilbert, MBio 6, e02527-14 (2015)

[37] A.R. Borneman, I.S. Pretorius, P.J. Chambers, Curr. Opin. Biotechnol. 24, 192-199 (2013)

[38] C.P. Kurtzman, C.J. Robnett, J. Clin. Microbiol. 35, 1216-1223 (1997)

[39] L. Oro, M. Ciani, F. Comitini, J. Appl. Microbiol. 116, 1209-1217 (2014)

[40] S.Y. Sun, H.S. Gong, X.M. Jiang, Y.P. Zhao, Food Microbiol. 44, 15-23 (2014)

[41] M. Gobbi, F. Comitini, P. Domizio, C. Romani, L. Lencioni, I. Mannazzu, M. Ciani, Food Microbiol. 33, 271-281 (2013)

[42] M. Quirós, V. Rojas, R. Gonzalez, P. Morales, Int. J. Food Microbiol. 181, 85-91 (2014)

[43] P.M. Izquierdo-Cañas, E. García-Romero, J.M. Heras-Manso, M. Fernández-González, Eur. Food Res. Technol. 239, 279-286 (2014)

[44] M. Azzolini, B. Fedrizzi, E. Tosi, F. Finato, P. Vagnoli, C. Scrinzi, G. Zapparoli Eur. Food Res. Technol. 235, 303-313 (2012)

[45] J. Marais, S. Afr. J. Enol. Vitic. 4, 49-58 (1983)
[46] S. Maicas, J.J. Mateo, Appl. Microbiol. Biotechnol. 67, 322-335 (2005)

[47] J.J. Mateo, R. Di Stefano, Food Microbiol. 14, 583-591 (1998)

[48] J.F. Ubeda-Iranzo, A.I. Briones-Perez, P.M. Izquierdo-Cañas, Food Microbiol. 15, 399-406 (1998)

[49] P. Van Rensburg, T. Stidwell, M.G. Lambrechts, R.R. Cordero-Otero, I.S. Pretorius, Anal Microbiol. 55, 33-42 (2005)

[50] A. Mendes-Ferreira, M.C. Clímaco, A. MendesFaia, J. Appl. Microbiol. 91, 67-71 (2001)

[51] M. Fernández-González, R. Di Stefano, A. Briones, Food Microbiol. 20, 35-41 (2003)

[52] T. Tominaga, C. Peyrot des Gachons, D. Dubourdieu, J. Agric. Food Chem. 46, 5215-5219 (1998)

[53] M.L. Murat, I. Masneuf, P. Darriet, V. Lavigne, T. Tominaga, D. Dubourdieu, Am. J. Enol. Vitic. 52, 136-139 (2001)

[54] J.H. Swiegers, I.S. Pretorius, Adv. Appl. Microbiol. 57, 131-175 (2005)

[55] C. Lang, H. Dornenburg, Appl. Microbiol. Biotechnol. 53, 366-75 (2000)

[56] E.F. Torres, T.V. Sepulveda, G.V. Gonzalez, Food Technol. Biotechnol. 44, 221-227 (2006)

[57] M. Maragon, S.C. Van Sluyter, E. Robinson, R. Muhlac, H. Holt, P.A. Haynes, P. Godden, P. Smith, E. Waters, Food Chem. 135, 1157-1165 (2012)

[58] C. Brandam, Q.P. Lai, A. Julien-Ortiz, P. Taillander, Biosci. Biotechnol. Biochem. 88, 1848-1853 (2013)

[59] P. Taillander, Q.P. Lai, A. Julien-Ortiz, C. Brandam, World J. Microbiol. Biotechnol. 30, 1959-1967 (2014)

[60] L.F. Bisson, R.E. Kunkee, Microbial interactions during wine production. In, Zeikus JG, Johnson EA (eds) Mixed cultures in biotechnology. (McGrawHill Inc., New York, 1991)

[61] M.E. Rodríguez, C.A. Lopes, R.J. Barbagelata, N.B. Barda, A.C. Caballero, Int. J. Food Microbiol. 138, 19-25 (2010)

[62] S. Sahay, B. Hamid, P. Singh, K. Ranjan, D. Chauhan, R.S. Rana and V.K. Chaurse, Lett. Appl. Microbiol. 57, 115-121 (2013) 